\title{
ERGODIC AND MIXING PROPERTIES OF EQUILIBRIUM MEASURES FOR MARKOV PROCESSES
}

\author{
ENRIQUE D. ANDJEL
}

\begin{abstract}
Let $S(t)$ be the semigroup corresponding to a Markov process on a metric space $X$. Suppose $S(t)$ commutes with a homeomorphism $T$ of $X$. We prove that under certain conditions, an equilibrium measure for the process is ergodic under $T$. We also show that, under stronger conditions this measure must be mixing under $T$. Several applications of these results are given.
\end{abstract}

\section{INTRODUCTION AND STATEMENT OF RESULTS}

This paper was motivated by two questions raised in the area of interacting particle systems by Liggett [12]. These questions ask whether or not certain equilibrium measures of translation invariant particle systems are ergodic. Our main result answers these questions affirmatively, but, since its proof is applicable to other systems we will state it in a more general context. To do so we start giving the necessary definitions.

Let $X$ be a metric space and $C=C(X)$ the Banach space of all bounded continuous real-valued functions on $X$, with the following norm:

$$
\|f\|=\sup _{\eta \in X}|f(\eta)| .
$$

Denote by $\mathscr{M}$ the set of all Borel probability measures on $X . \mathscr{M}$ will be endowed with the topology of weak convergence and its Borel $\sigma$-algebra. A homeomorphism $T$ on $X$ induces transformations $T^{*}$ and $T^{* *}$ on $C$ and respectively, through the following equalities:

$$
\left(T^{*} f\right)(\eta)=f(T \eta) \quad \forall f \in C, \forall \eta \in X,
$$

and

$$
\int f d\left(\mu T^{* *}\right)=\int\left(T^{*} f\right) d \mu \quad \forall f \in C, \forall \mu \in \mathscr{M} .
$$

In the sequel we will drop the superscripts of $T^{*}$ and $T^{* *}$ because no confusion will occur.

Now, let $\left\{T_{i}\right\}_{i \in I}$ be a collection of homeomorphisms of $X$, indexed by a finite set $I$, such that

$$
T_{i} T_{j}=T_{j} T_{i} \quad \forall i, j \in I .
$$

Received by the editors March 23, 1988 and, in revised form, July 5, 1988.

1980 Mathematics Subject Classification (1985 Revision). Primary 60K35; Secondary 60J25.

Key words and phrases. Markov process, interacting particle system, ergodic, mixing. 
By a Feller semigroup $\{S(t), t \geq 0\}$ on $C$ we mean

(a) $\forall t \geq 0, S(t)$ is a linear operator on $C$,

(b) $S(0)$ is the identity on $C$,

(c) $S(t+s)=S(t) S(s) \quad \forall t, s \geq 0$,

(d) $S(t) 1=1 \quad \forall t \geq 0$ where 1 is the function $f(\eta) \equiv 1$,

(e) $S(t) f \geq 0 \quad \forall f \geq 0$ in $C$ and all $t \geq 0$,

(f) $\forall t \geq 0 \forall \mu \in \mathscr{M}$ there exists $\mu S(t) \in \mathscr{M}$ such that

$$
\int f d \mu S(t)=\int S(t) f d \mu \quad \forall f \in C .
$$

Note that this is not the usual definition of a Feller semigroup, since we do not require $X$ to be locally compact and $S(t)$ to be strongly continuous (see page 166 of [6]). It will prove, however, to be convenient for our purposes. Note also that it follows from (a), (d) and (e) that for all $t \geq 0, S(t)$ is a continuous operator of norm 1. Finally observe that the uniqueness of the measure $\mu S(t)$ in (f) follows from Proposition 4.2 on page 111 of [6] and the Dominated Convergence Theorem. The existence of $\mu S(t)$ is a consequence of the Riesz Representation Theorem and the continuity of $S(t)$, if $X$ is compact. In view of the uniqueness of $\mu S(t)$ and of c) and $\mathrm{f})$, we may also consider $S(t)$ as a semigroup acting on $\mathscr{M}$ (i.e. $\mu S(t+s)=\mu S(t) S(s))$. Since $S(t) f \in C$ for all $f \in C, S(t)$ acts continuously on $\mathscr{M}$ too.

In this paper we will consider Feller semigroups $S(t)$ satisfying

$$
S(t) T_{i}=T_{i} S(t) \quad \forall i \in I, \forall t \geq 0 .
$$

The subsets of $\mathscr{M}$ given by $\left\{\mu: \mu T_{i}=\mu \quad \forall i \in I\right\}$ and $\{\mu: \mu S(t)=\mu$ $\forall t \geq 0\}$ will be denoted by $\mathscr{S}$ and $\mathscr{I}$ respectively. Besides this, if $\mathscr{A} \subset \mathscr{M}$, then $\mathscr{A}_{e}$ will be the set of extreme points of $\mathscr{A}$.

An element $\mu \in \mathscr{S}$ will be said to be $T_{1}$-ergodic if any Borel measurable real-valued function $f$ on $X$, such that $T_{i} f=f \mu$-a.e. $\forall i \in I$, is $\mu$-a.e. constant.

Since we will deal with multi-indexed sequences, it is convenient to introduce the following notation: $k_{I}$ and $n_{I}$ will be sequences of nonnegative integers indexed by $I$ (i.e $\left.k_{I}=\left(k_{i}\right)_{i \in I}\right)$, expressions as $k_{I} \leq n_{I}$ or $n_{I} \rightarrow \infty$ will be interpreted componentwise and $\left|n_{I}\right|, \prod n_{I}$ and $T^{k_{l}}$ will denote $\sum_{i \in I} n_{i}$, $\prod_{i \in I}\left(n_{i}+1\right)$ and $\prod_{i \in I} T_{i}^{k_{i}}$ respectively.

If $\mu \in \mathscr{S}$, then $T_{i}$ can be extended to $L^{2}(\mu)$ by means of $\left(T_{i} f\right)(\eta)=f\left(T_{i} \eta\right)$ $\forall \eta \in X, \forall f \in L^{2}$, and it is well known that the following three conditions are equivalent:

(1) $\mu$ is $T_{I}$-ergodic,

(2) $\lim _{n_{l} \rightarrow \infty}\left(1 / \prod n_{I}\right) \sum_{k_{l} \leq n_{l}} \int f\left(T^{k_{l}} g\right) d \mu=\int f d \mu \cdot \int g d \mu \forall f, g \in L^{2}(\mu)$,

(3) $\mu \in \mathscr{S}_{e}$.

For a proof of these equivalences the reader is referred to Chapter I of [12]. 
The proofs of Proposition 4.11 and Corollary 4.14 in that chapter can be easily adapted to the present situation.

Let $\nu \in \mathscr{S}$ and suppose there exists a Borel probability measure $\lambda$ on $\mathscr{S}_{e}$, such that

$$
\nu=\int_{\mathscr{P}_{e}} \mu d \lambda(\mu)
$$

This is called an ergodic decomposition of $\nu$. The existence (and uniqueness) of such a decomposition for all elements of $\mathscr{S}$ follows from Theorems 3.1 and 6.1 of [5], if $X$ is a complete separable metric space.

An element $\mu \in \mathscr{S}$ is called $T_{I}$-mixing if

$$
\lim _{\left|n_{l}\right| \rightarrow \infty} \int f\left(T^{n_{l}} g\right) d \mu=\int f d \mu \int g d \mu \quad \forall f, g \in L^{2}(\mu)
$$

where the $T_{i}$ 's have been extended as before to $L^{2}(\mu)$. Obviously if $\mu$ is $T_{I}$-mixing, then it is $T_{I}$-ergodic.

Suppose $S(t)$ and $\left\{T_{i}\right\}$ are as above and $\mu$ is an element of $\mathscr{I} \cap \mathscr{S}$. When is it the case that $\mu$ is $T_{I}$-ergodic? When is it $T_{I}$-mixing? Theorem (1.4) below gives sufficient conditions for the answers to be affirmative. In its statement, as well as in the sequel, $\delta_{\eta}$ will denote the Dirac measure on $\eta$.

Theorem (1.4). Let $X$ be a metric space, $S(t)$ a Feller semigroup on $C(X)$ and $\left\{T_{i}\right\}_{i \in I}$ a finite collection of homeomorphisms on $X$ satisfying (1.1) and (1.2). Suppose $\nu \in \mathscr{I} \cap \mathscr{S}$ and $B$ is a subset of $C(X)$ which is dense in $L^{2}(\nu)$. Moreover assume that

$$
\lim _{\left|n_{l}\right| \rightarrow \infty} \int\left|S(t)\left[f T^{n_{l}} g\right]-S(t) f S(t)\left(T^{n_{l}} g\right)\right| d \nu=0 \quad \forall f, g \in B .
$$

(i) If $\nu=\int \mu d \lambda(\mu)$ is an ergodic decomposition of $\nu$ and there exists a sequence $t_{n} \uparrow \infty$, such that $\lim _{n} \int f d \mu S\left(t_{n}\right)=\int f d \nu \forall f \in B$ and $\lambda$-almost all $\mu$, then $\nu$ is $T_{I}$-ergodic.

(ii) If there exists a sequence $t_{n} \uparrow \infty$, such that $\lim _{n} \int f d\left(\delta_{\eta} S\left(t_{n}\right)\right)=\int f d \nu$ $\forall f \in B$ and $\nu$-almost all $\eta$, then $\nu$ is $T_{I}$-mixing.

In $\S 2$ we prove Theorem (1.4). $\S \S 3-6$ are applications of that theorem. These applications can be read in any order, except for the fact that Proposition (4.1) is needed in the first part of $\S 5 . \S 7$ contains examples to show that one cannot drop any of the hypotheses of Theorem (1.4). Some open problems are also stated in $\S 7$. In $\S \S 3-7, X$ will be a translation invariant subspace of $W^{\mathbf{Z}^{d}}$, where $W$ is a metric space, $\mathbf{Z}^{d}$ is the $d$-dimensional integer lattice and the $T_{i}$ 's will be the operators on $X$ induced by the translations on $Z^{d}$. Since the 
$T_{i}$ 's are fixed in those sections, we will write that an element $\mu$ of $\mathscr{S}$ is ergodic or mixing instead of $T_{I}$-ergodic or $T_{I}$-mixing respectively.

2. Proof of Theorem (1.4)

Let $f, g \in B$ and $\mu \in \mathscr{M}$. Then define

$$
A\left(n_{I}, f, g, \mu\right)=\int f T^{N_{I}} g d \mu
$$

and

$$
B\left(n_{I}, f, g, \mu\right)=\frac{1}{\prod n_{I}} \sum_{k_{I} \leq n_{I}} A\left(k_{I}, f, g, \mu\right) .
$$

To prove part (i) write

$$
\begin{aligned}
B\left(n_{I}, f, g, \nu\right) & =B\left(n_{I}, f, g, \nu S(t)\right) \\
& =\frac{1}{\prod n_{I}} \sum_{k_{l} \leq n_{I}} \int\left[\int f T^{k_{l}} g d \mu S(t)\right] d \lambda(\mu) .
\end{aligned}
$$

Since

$$
\begin{aligned}
\int f T^{k_{l}} g d \mu S(t)= & \int S(t)\left[f T^{k_{l}} g\right] d \mu \\
= & \int S(t) f T^{k_{l}}[S(t) g] d \mu \\
& +\int\left(S(t)\left[f T^{k_{l}} g\right]-S(t) f T^{k_{l}}(S(t) g)\right) d \mu,
\end{aligned}
$$

we obtain the following equality:

$$
\begin{aligned}
B\left(n_{I}, f, g, \nu\right)= & \int\left[\frac{1}{\prod n_{I}} \sum_{k_{l} \leq n_{I}} \int S(t) f T^{k_{I}}(S(t) g) d \mu\right] d \lambda(\mu) \\
& +\frac{1}{\prod n_{I}} \sum_{k_{I} \leq n_{I}} \int\left(S(t)\left[f T^{k_{I}} g\right]-S(t) f T^{k_{I}}(S(t) g)\right) d \nu .
\end{aligned}
$$

It now follows from (1.5) and the fact that $\mu$ is $T_{I}$-ergodic for $\lambda$-almost all $\mu$, that

$$
\lim _{n_{I} \rightarrow \infty} B\left(n_{I}, f, g, \nu\right)=\int\left[\int S(t) f d \mu \cdot \int S(t) g d \mu\right] d \lambda(\mu) \quad \forall t \geq 0 .
$$

Taking limits in (2.1) along the sequence $t_{n}$ given by part (i) of Theorem (1.4) and using the Dominated Convergence Theorem we get

$$
\lim _{n_{I}} B\left(n_{I}, f, g, \nu\right)=\int f d \nu \int g d \nu \quad \forall f, g \in B .
$$

Since $B$ is dense in $L^{2}(\nu)$, the same equality holds for all $f, g \in L^{2}(\nu)$. Hence, $\nu$ is $T_{I}$-ergodic. 
For (ii) write for $t \geq 0$

$$
\begin{aligned}
A\left(n_{I}, f\right. & , g, \nu)=A\left(n_{I}, f, g, \nu S(t)\right) \\
= & \int\left[S(t)\left(f T^{n_{I}} g\right)(\eta)-S(t) f(\eta) S(t)\left(T^{n_{I}} g\right)(\eta)\right] d \nu(\eta) \\
& +\int S(t) f(\eta)\left[S(t)\left(T^{n_{I}} g\right)(\eta)-\int g(\xi) d \nu(\xi)\right] d \nu(\eta) \\
& +\int S(t) f(\eta) d \nu(\eta) \cdot \int g(\xi) d \nu(\xi) .
\end{aligned}
$$

The absolute value of the second term of the right-hand side is bounded above by

$$
\|f\| \int\left|S(t)\left(T^{n}{ }^{n} g\right)(\eta)-\int g(\xi) d \nu(\xi)\right| d \nu(\eta) .
$$

It now follows from the invariance of $\nu$ and $S(t)$ under $T^{n_{l}}$ that this bound is equal to

$$
\|f\| \int\left|S(t) g(\eta)-\int g(\xi) d \nu(\xi)\right| d \nu(\eta) .
$$

Note that this bound is independent of $\eta_{I}$ and that it converges to 0 if $t$ goes to infinity along the sequence $t_{n}$ given in part (ii) of Theorem (1.4). Therefore, given $\varepsilon>0$ there exists an integer $n_{0}$ such that for all $n \geq n_{0}$

$$
\begin{aligned}
& \limsup _{n_{l} \rightarrow \infty} A\left(n_{I}, f, g, \nu\right) \\
& \quad \leq \limsup _{n_{I} \rightarrow \infty} \int\left[S\left(t_{n}\right)\left(f T^{n_{l}} g\right)(\eta)-S\left(t_{n}\right) f(\eta) S\left(t_{n}\right)\left(T^{n_{l}} g\right)(\eta)\right] d \nu(\eta) \\
& \quad+\int S\left(t_{n}\right) f d \nu(\eta) \int g(\xi) d \nu(\xi)+\varepsilon .
\end{aligned}
$$

Using (1.5) we see that the first term of the right-hand side above is 0 . Hence,

$$
\begin{aligned}
\limsup _{n_{1} \rightarrow \infty} A\left(n_{I}, f, g, \nu\right) & \leq \lim _{n} \int S\left(t_{n}\right) f d \nu(\eta) \cdot \int g(\xi) d \nu(\xi)+\varepsilon \\
& =\int f d \nu \cdot \int g d \nu+\varepsilon .
\end{aligned}
$$

A similar argument shows that

$$
\liminf _{n_{I} \rightarrow \infty} A\left(n_{I}, f, g, \nu\right) \geq \int f d \nu \int g d \nu-\varepsilon .
$$

Since $\varepsilon$ is arbitrary we have $\forall f, g \in B$

$$
\lim _{n_{I} \rightarrow \infty} A\left(n_{I}, f, g, \nu\right)=\int f d \nu \int g d \nu
$$

Using the fact that $B$ is dense in $L^{2}(\nu)$, we see that the same equality holds for all $f, g \in L^{2}(\nu)$. This completes the proof of part (ii).

\section{ERGODIC INTERACTING PARTICLE SYSTEMS}

In this section we consider the Markov processes constructed in $§ I-3$ of [12]. These processes have as state space $X=W^{S}$, where $W$ is a compact metric 
space and $S$ is a finite or countable set. The space $X$, with the product topology, becomes a compact metric space. To describe the processes we quote [12]: "The local dynamics of the system are described by a collection of transition measures $c_{T}(\eta, d \zeta)$. For each $\eta \in X$ and finite $T \subset S, c_{T}(\eta, d \zeta)$ is assumed to be a finite positive measure on $W^{T}$. We will assume further that for each $T$ the mapping $\eta \rightarrow c_{T}(\eta, d \zeta)$ is continuous from $X$ to the space of finite measures on $W^{T}$, with the topology of weak convergence. The interpretation is that $\eta$ is the current configuration of the system, $c_{T}\left(\eta, W^{T}\right)$ is the rate at which a transition will occur involving the coordinates in $T$, and $c_{T}(\eta, d \zeta) / c_{T}\left(\eta, W^{T}\right)$ is the distribution of the restriction to $T$ of the new configuration after that transition has taken place." In this application $S$ will be the $d$-dimensional integer lattice $\mathbf{Z}^{d}$ and the local dynamics of the processes will be invariant under translations on $\mathbf{Z}^{d}$. Then, as shown in [12], under the assumptions

$$
\sum_{K \ni 0} \sup _{\eta \in X}\left\{c_{K}\left(\eta, W^{K}\right)\right\}<\infty
$$

and

$$
\sum_{K \ni 0} \sum_{u \neq 0} \sup \left\{\left\|c_{K}\left(\eta_{1}, d \xi\right)-c_{K}\left(\eta_{2}, d \xi\right)\right\|_{K}: \eta_{1}(y)=\eta_{2}(y) \text { for all } y \neq u\right\}<\infty
$$

$\left(\|\cdot\|_{K}\right.$ being the total variation norm of a measure on $\left.W^{K}\right)$, there exists a Feller semigroup $\{S(t), t \geq 0\}$, acting on $C(X)$ whose generator corresponds to the dynamics described above. Since this generator is invariant under translations, it follows from part b) by Theorem 2.9 of Chapter I of [12] that the semigroup $S(t)$ shares this property. Moreover, under the further assumption

$$
\sum_{K \ni 0}|K| \sup _{n \in X}\left\{C_{K}\left(\eta, W^{K}\right)\right\}<\infty
$$

$(|\cdot|$ denoting the cardinality of a set), it follows from Theorem 4.6 on pages 35-36 of [12] that (1.5) holds for all continuous $f$ and $g$ depending on finitely many coordinates.

An interacting particle system on $X$ is called ergodic if there exists a (necessarily unique) measure $\nu \in \mathscr{M}$ such that $\lim _{t \rightarrow \infty} \mu S(t)=\nu$ for all $\mu \in \mathscr{M}$. From these definitions, the above considerations and part (ii) of Theorem (1.4) we conclude that:

If a translation invariant particle system on $W^{Z^{d}}$ satisfies (3.3) and is ergodic, then its unique invariant measure is mixing.

Since spin flip systems, as defied in Chapter III of [12], are special cases of these processes for which (3.3) follows from (3.1), this answers affirmatively the question raised in Problem 7 of that chapter.

For certain interacting particle systems, it is easier to show the convergence of $\mu S(t)$ if one assumes that $\mu \in \mathscr{S}$. In these cases part (i) of Theorem (1.4) 
may be applicable. In particular if $\mathscr{I} \cap \mathscr{S}=\{\nu\}$ and $\mu S(t)$ converges for all $\mu \in \mathscr{S}$, then $\nu$ must be ergodic.

\section{SySTEMS OF STOCHASTIC DIFFERENTIAL EQUATIONS}

In this section we apply part (i) of Theorem (1.4) to the processes studied by Holley and Stroock in [11]. To do so we need the following proposition which will be used in the next section too. In the statement of the proposition $\mathscr{A}$ is a subset of $\mathscr{M}$ and $X$ and $\mathscr{M}$ are as in the introduction. Although we do not require $\mathscr{A}$ to be convex, we will only apply the proposition in situations in which it is.

Proposition (4.1). Suppose $X$ and $\mathscr{A}$ are compact. Let $\lambda$ be a Borel probability measure on $\mathscr{M}, \nu$ an extreme point in $\mathscr{A}$ and $K_{n}: \mathscr{M} \rightarrow \mathscr{M}$ a sequence of measurable maps such that $\int K_{n}(\mu) d \lambda(\mu)=\nu$ for all $n$. If for $\lambda$-almost all $\mu$ all weak limits of $K_{n}(\mu)$ belong to $\mathscr{A}$, then there exists a sequence $n_{k} \uparrow \infty$ satisfying $\lim _{k} K_{n_{k}}(\mu)=\nu$ for $\lambda$-almost all $\mu$.

Proof. In this proof statements about convergence in probability and almost sure convergence are related to the probability space $(\mathscr{M}, \lambda)$.

Let $\lambda_{n}$ be the measure induced by $\lambda$ under the mapping $K_{n}$. The assumptions of the proposition immediately imply that $\int \mu d \lambda_{n}=\nu$ and that all weak limits of $\lambda_{n}$ concentrate on $\mathscr{A}$. Therefore, if $\gamma$ is a weak limit of $\lambda_{n}$, then $\int \mu d \gamma=\nu$. Since $\nu$ is extreme, $\gamma=\delta_{\nu}$. Therefore $\lim _{n} \lambda_{n}=\delta_{\nu}$ (weakly). This implies that $K_{n}$ converges to the constant $\nu$ in probability. Hence, there exists a subsequence of $K_{n}$ converging to $\nu$ almost surely.

In the final part of this section we will assume the reader to be familiar with the contents of [11]. The processes studied in $\S \S 4$ and 5 of that paper have as state space $T^{\mathbf{Z}^{d}}$, where $T=\{z \in \mathbf{C}:|z|=1\}$. It follows from the construction of these processes, more precisely from Lemma (1.2) and Theorem (1.3) of [11], that for all pairs of continuous real-valued functions $f$ and $g$, depending on finitely many coordinates, we have

$$
\lim _{\left|n_{1}\right| \rightarrow \infty}\left\|S(t)\left(f T^{n_{1}} g\right)-S(t) f S(t)\left(T^{n_{1}} g\right)\right\|=0 .
$$

Hence (1.5) is satisfied for all $\nu \in \mathscr{M}$. The fact that $S(t)$ commutes with the translations of $Z^{d}$ follows also from the construction, as explained in [11]. Finally the hypothesis for part (i) of Theorem (1.4) is a consequence of Proposition (4.1) in this paper and Theorem (4.35) of [11]. Therefore, for the processes studied in $\S 4$ of [11] we conclude that all elements of $(\mathscr{G} \cap \mathscr{S})_{e}$ are ergodic where $\mathscr{G}$ is the set of Gibbs states.

Remark. As explained at the beginning of $\S 4$ of [11] all Gibbs states are reversible for the process. Therefore $\mathscr{G} \subset \mathscr{F}$. Corollary (4.37) of [11] states that $\mathscr{I} \cap \mathscr{S} \subset \mathscr{G}$. Hence $(\mathscr{I} \cap \mathscr{S})_{e}=(\mathscr{G} \cap \mathscr{S})_{e}$. 


\section{Stochastic Ising models, Gibbs states}

In this section we apply Theorem (1.4) to stochastic Ising models, as defined in Chapter IV of [12]. We will assume that the reader is familiar with the notation of that chapter.

Given a translation invariant potential $\left\{J_{R}\right\}$ on $\mathbf{Z}^{d}$, such that

$$
\sum_{R \ni 0}|R|\left|J_{R}\right|<\infty
$$

the corresponding stochastic Ising model satisfies the hypothesis of Theorem (4.6) of Chapter I of [12]. The conclusion of that theorem shows that (1.5) is satisfied for all cylinder functions $f$ and $g$ and all $\nu \in \mathscr{M}$. To see that the hypothesis of part i) of Theorem (1.4) is fulfilled too, we appeal to Proposition (4.1) and to the fact that all weak limits of $\mu S(t) \quad(\mu \in \mathscr{S})$ belong to $\mathscr{G}$. This last statement was proved by Holley [10] for finite range potentials, and extended to potentials satisfying (5.1) by Higuchi and Shiga [9]. These considerations show that all elements of $(\mathscr{G} \cap \mathscr{S})_{e}$ are ergodic where $\mathscr{G}$ denotes the set of Gibbs states.

We will show in $\S 7$ that elements of $(\mathscr{G} \cap \mathscr{S})_{e}$ may not be mixing. We do, however, have the following result: all elements of $\mathscr{G}_{e} \cap \mathscr{S}$ are mixing. To prove this, take a countable set of cylinder functions $\left\{f_{m}\right\}$, dense in $C(X)$, then apply Theorem (4.13) in Chapter IV of [12] and the diagonal argument, to obtain a sequence $t_{n}$ diverging to infinity and satisfying

$$
\lim _{n} S\left(t_{n}\right) f_{m}(\eta)=\int f_{m} d \nu \quad \nu \text {-a.e. } \forall m
$$

Then apply part (ii) of Theorem (1.4).

Remarks. As in the previous section $\mathscr{G} \cap \mathscr{S}=\mathscr{I} \cap \mathscr{S}$. This follows from the results of Holley, extended by Higuchi and Shiga, which were mentioned above.

The second result in this section is not new. A direct proof, not recurring to stochastic Ising models, can be found in $[14$, p. 20]. Better results are known when Dobrushin's uniqueness condition is satisfied. This condition implies that $\mathscr{G}$ is a singleton, and that its unique element has exponentially decaying correlations (see [2 and 3]). For a more recent treatment of the decay of the correlations under that condition, we refer the reader to [7]. The reader is also referred to [4], where for each finite $V \subset \mathbf{Z}^{d}$, a condition $C_{V}$ is given. It is then proved there, that if $C_{V}$ is satisfied for some $V$, then there is a unique Gibbs state and its correlations decay exponentially. This generalizes the above results, because $C_{V}$. coincides with the previous condition when $V$ is a singleton.

\section{LINEAR SYSTEMS ON $[0, \infty)^{\mathbf{Z}^{d}}$}

These processes evolve on appropriate subspaces of $[0, \infty)^{S}$, for some countable set $S$. They are called linear because given one $\eta_{t}$, there exists a family $\left\{A_{t}(x, y), t \geq 0, x, y \in S\right\}$ of nonnegative random matrices such that 
$\eta_{t}(x)=\sum_{y \in S} A_{t}(x, y) \eta_{0}(y)$. The main reference to these systems is Chapter IX of [12] and we assume that the reader is familiar with, at least, the first section of that chapter. Besides the assumptions made in that section, we will only consider cases in which $S=\mathbf{Z}^{d}$ and the formal generator of the process is invariant under translations on $\mathbf{Z}^{d}$. Under these hypotheses it is shown in $\S 2$ of Chapter IX of [12] that $\delta_{\beta} S(t)$ converges, as $t$ goes to infinity ( $\beta$ denoting the configuration $\eta(x) \equiv \beta)$. The limit is called $\nu_{\beta}$, belongs to $\mathscr{S}$ and, as proved in [12], either it is $\delta_{0}$ or it satisfies $\int \eta(x) d \nu_{\beta}=\beta$. In this section we prove that $\nu_{\beta}$ is ergodic, thus solving Problem 4 on page 468 of [12]. Since the case in which $\nu_{\beta}=\delta_{0}$ is trivial, we assume from now on that $\int \eta(x) d \nu_{\beta}=\beta>0$.

Given one of the above processes, the subspace $X$ of $[0, \infty)^{\mathbf{Z}^{d}}$ on which it is defined is determined in [12], in the following way: Lemma 1.6 on page 423 of [12] constructs a function $\alpha: \mathbf{Z}^{d} \rightarrow \mathbf{R}_{+}$, then $X$ is defined as

$$
\left\{\eta \in[0, \infty)^{\mathbf{Z}^{d}}: \sum_{x \in \mathbf{Z}^{d}} \eta(x) \alpha(x)<\infty\right\} .
$$

The topology of $X$ is now determined by means of the following "norm":

$$
\|\eta-\xi\|=\sum_{x \in \mathbf{Z}^{d}}|\eta(x)-\xi(x)| \alpha(x) .
$$

In this way, $X$ becomes a complete separable metric space.

Let $\mathscr{L}$ be the subset of $C(X)$ formed by the functions $f$ for which there exists a constant $L$ such that $|f(\eta)-f(\xi)| \leq L\|\eta-\xi\| \forall \eta, \xi \in X$. Given $f \in$ $\mathscr{L}$, call $L(f)$ the smallest constant satisfying this inequality. Fix a sequence of finite subsets $S_{n}$ of $\mathbf{Z}^{d}$, such that $S_{n} \uparrow \mathbf{Z}^{d}$, and call $\mathscr{L}_{n}$ the subset of elements of $\mathscr{L}$ not depending on coordinates off $S_{n}$.

Remark. Here we have departed a little from the notation of [12]. There, elements of $\mathscr{L}$ have continuous partial derivatives and may be unbounded.

There are, however, no obstacles to the construction of a semigroup $S(t)$ on $\mathscr{L}$, as it is done in [12]. We make now five assertions:

(1) By appropriately choosing the function $\alpha, X$ can be made to be invariant under translations.

(2) The semigroup $S(t)$ can be extended to $C(X)$ and the extension is a Feller semigroup as defined in the introduction.

(3) The extended semigroup commutes with translations on $\mathbf{Z}^{d}$.

(4) If $\nu \in \mathscr{S}$ and $B=\bigcup_{n=1}^{\infty} \mathscr{L}_{n}$ then (1.5) is satisfied.

(5) $B$ is dense in $L^{2}(\nu)$.

The proofs of the first four assertions depend heavily on the construction of the process and are quite unrelated to the rest of this paper. These proofs do not differ substantially from proofs of similar assertions that have been made for other processes. The reader should, however, note that a result as strong as 
Theorem (4.6) in Chapter I of [12], does not hold in the present context. Nevertheless, the proof of the fourth assertion can be done by means of standard coupling techniques and the use of some of the ideas appearing in the construction of these linear systems. The proof of the last assertion is standard. For these reasons, all these proofs will be omitted.

Since $X$ is a complete separable metric space and $\nu_{\beta} \in \mathscr{S}$, there is a unique ergodic decomposition

$$
\nu_{\beta}=\int \mu d \lambda(\mu)
$$

The ergodicity of $\nu_{\beta}$ follows now from Lemma (6.1) below and part (i) of Theorem (1.4). The proof of Lemma (6.1) is an adaptation of the proof of Lemma 2.9 on page 436 of [12].

Lemma (6.1). There exists a sequence $t_{n} \uparrow \infty$ such that $\int f d \mu S\left(t_{n}\right)$ converges to $\int f d \nu_{\beta}$ for all $f \in B$ and $\lambda$-almost all $\mu$.

Proof. For $\mu \in \mathscr{S}$, define $\varphi(\mu)=\int \eta(x) d \mu$. In view of our assumption concerning $\nu_{\beta}$ we have

$$
\int \varphi(\mu) d \lambda(\mu)=\beta
$$

Let $\xi$ be an element of $[0, \infty)^{Z^{d}}$, different from 0 and with finite support. From Jensen's inequality we obtain

$$
\exp \left[-\varphi(\mu) \sum \xi(x)\right] \leq \int \exp \left[-\sum \eta(x) \xi(x)\right] d \mu
$$

(In this proof all sums are over $\mathbf{Z}^{d}$ and the integrated variable is $\eta$, unless it is otherwise specified.) Let $\xi_{t}$ be the dual process of $\eta_{t}$ (see pages 430-431 of [12]). Then

$$
E^{\xi}\left[\exp \left(-\varphi(\mu) \sum \xi_{t}(x)\right)\right] \leq \int E^{\xi}\left[\exp \left(-\sum \eta(x) \xi_{t}(x)\right)\right] d \mu .
$$

By Theorem 1.25 on page 431 of [12], this last inequality can be written as

$$
\begin{array}{r}
\int \exp \left[-\frac{\varphi(\mu)}{\beta} \sum \eta(x) \xi(x)\right] d \delta_{\beta} S(t) \\
\leq \int \exp \left[-\sum \eta(x) \xi(x)\right] d \mu S(t)
\end{array}
$$

For $\gamma \geq 0$ define

$$
\Phi_{\xi}(\gamma)=\int \exp \left[-\gamma \sum \eta(x) \xi(x)\right] d \nu_{\beta}
$$

Then

$$
\Phi_{\xi}\left(\frac{\varphi(\mu)}{\beta}\right)-\int \exp \left[-\frac{\varphi(\mu)}{\beta} \sum \eta(x) \xi(x)\right] \delta_{\beta} S(t) \rightarrow 0 \quad \text { as } t \rightarrow \infty .
$$

Therefore, by (6.2)

$$
\Phi_{\xi}\left(\frac{\varphi(\mu)}{\beta}\right) \leq \int \exp \left[-\sum \eta(x) \xi(x)\right] d \mu S(t)+f(\mu, t)
$$


where

$$
|f(\mu, t)| \leq 1 \quad \forall \mu, t, \quad \text { and } \quad \lim _{t} f(\mu, t)=0 \quad \forall \mu
$$

Hence

$$
\int \Phi_{\xi}\left(\frac{\varphi(\mu)}{\beta}\right) d \lambda(\mu) \leq \Phi_{\xi}(1)+\int f(\mu, t) d \lambda(\mu) .
$$

Letting $t \rightarrow \infty$, it follows from (6.4) and the Dominated Convergence Theorem that

$$
\int \Phi_{\xi}\left(\frac{\varphi(\mu)}{\beta}\right) d \lambda(\mu) \leq \Phi_{\xi}(1) .
$$

Since $\Phi_{\xi}$ is strictly convex, this implies that

$$
\varphi(\mu)=\beta \quad \lambda \text {-a.e }
$$

Integrate (6.3) with respect to $\lambda$; in view of (6.5) this yields

$$
\begin{aligned}
\Phi_{\xi}(1) & \leq \int\left[\int \exp \left[-\sum \eta(x) \xi(x)\right] d \mu S(t)+f(\mu, t)\right] d \lambda(\mu) \\
& =\Phi_{\xi}(1)+\int f(\mu, t) d \lambda(\mu) .
\end{aligned}
$$

By (6.4) and the Dominated Convergence Theorem the limit as $t \rightarrow \infty$ of the right-hand side above is $\Phi_{\xi}(1)$. From (6.3) and (6.5) we obtain

$$
\Phi_{\xi}(1) \leq \int \exp \left[-\sum \eta(x) \xi(x)\right] d \mu S(t)+f(\mu, t) \quad \lambda \text {-a.e. }
$$

Hence, there exists $h(\mu, t) \geq 0$ such that

$$
f(\mu, t)+\int \exp \left[-\sum \eta(x) \xi(x)\right] d \mu S(t)=\Phi_{\xi}(1)+h(\mu, t) \quad \lambda \text {-a.e. }
$$

Plugging this in (6.6) we get

$$
\Phi_{\xi}(1) \leq \Phi_{\xi}(1)+\int h(\mu, t) d \lambda(\mu) \leq \Phi_{\xi}(1)+\int f(\mu, t) d \lambda(\mu) .
$$

In view of the observation following (6.6), this implies that $h(\mu, t) \rightarrow 0$ in $L^{1}(\lambda)$. Hence, there exists a sequence $t_{n} \uparrow \infty$, such that

$$
h\left(\mu, t_{n}\right) \rightarrow 0 \quad \lambda \text {-a.e. }
$$

It follows from (6.4), (6.7) and (6.8) that

$$
\begin{aligned}
& \lim _{n \rightarrow \infty} \int \exp \left[-\sum \eta(x) \xi(x)\right] d \mu S\left(t_{n}\right) \\
& \quad=\Phi_{\xi}(1)=\int \exp \left[-\sum \eta(x) \xi(x)\right] d \nu_{\beta} \quad \lambda \text {-a.e. }
\end{aligned}
$$

Using the diagonal argument we obtain a further subsequence, also called $t_{n}$, for which (6.9) holds for all $\xi \not \equiv 0$ with rational coordinates and finite support. By monotonicity and continuity, (6.9) must then hold for all $\xi$ with finite support. Therefore, for $\lambda$-almost all $\mu$ the finite-dimensional Laplace transforms 
of $\mu S\left(t_{n}\right)$ converge to the corresponding finite-dimensional Laplace transforms of $\nu_{\beta}$. This proves the lemma.

Remark. In [12], $\alpha$ is, at a certain point, assumed to be symmetric. Under that assumption, the process $\eta_{t}$ and its dual $\xi_{t}$ have the same state space. Here, that assumption is not necessary, because the dual process is only needed on the set $\left\{\xi: \sum_{x \in \mathbf{Z}^{d}} \xi(x)<\infty\right\}$.

\section{COUNTEREXAMPLeS AND OPEN PROBLEMS}

We start this section with examples showing what can happen if the hypotheses of Theorem (1.4) are relaxed. We will not give complete proofs of our statements, but the interested reader will find no serious difficulty in providing them. We start with an example of a translation invariant process, satisfying (1.5) and possessing a unique invariant measure which is not ergodic.

Let $X=T^{\mathbf{Z}}$ where $T=\{z \in \mathbf{C}:|z|=1\}$. Consider a process on $X$ with the following dynamics:

(a) All coordinates of $\eta \in X$ turn counterclockwise at the same constant speed $v>0$.

(b) At times given by independent Poisson processes of parameter one, coordinates choose a neighbor independently of the Poisson processes. Each neighbor has probability $\frac{1}{2}$ of being chosen. Then the coordinate becomes equal to the chosen neighbor.

It is easy to verify that this process and the one-dimensional nearest neighbor voter model analyzed in Chapter $\mathrm{V}$ of [12], form blocs of equal coordinates in the same way. Then, by Corollary 1.13 on page 231 of [12], an invariant measure for any of these processes must concentrate on constant configurations. From this it is easy to deduce that the process we are considering has a unique invariant measure $\nu$. This measure is the only one concentrating on constant configurations and having as marginals the normalized Lebesgue measure on $T$. Of course, this measure is not ergodic.

For an example satisfying the hypothesis of part (i) of Theorem (1.4) but not satisfying the conclusion of part (ii) of that theorem, we refer the reader to [13]. It follows from the results in that paper that the nearest neighbor antivoter model in one dimension has as extremal invariant measures the point masses on

$$
\eta(x)= \begin{cases}1 & \text { if } x \text { is even } \\ 0 & \text { if } x \text { is odd }\end{cases}
$$

and on

$$
\xi(x)= \begin{cases}0 & \text { if } x \text { is even } \\ 1 & \text { if } x \text { is odd }\end{cases}
$$

Hence the unique element of $\mathscr{I} \cap \mathscr{S}$ is $\nu=\frac{1}{2}\left(\delta_{\eta}+\delta_{\xi}\right)$. It is also proved in [13] that $\lim _{t} \mu S(t)=\nu$ for all $\mu \in \mathscr{S}$. Obviously $\nu$ is ergodic but not mixing.

Another example of this kind, for which all the transition rates are strictly positive, is given by a nonattractive two-dimensional Ising model. Take as 
potential $J_{K}=\beta$ if $K=\{x, y\}$ with $\|x-y\|=1$ (Euclidean norm) and $J_{K}=0$ otherwise. For $\beta<0$ and $|\beta|$ sufficiently large, $\mathscr{G} \cap \mathscr{S}$ is a singleton. Its unique element is ergodic as proved in $\S 5$, but it is not mixing. This last assertion follows from the facts stated in the second paragraph on page 204 of [12] and the results of [1 and 8].

For an example of a translation invariant, ergodic process in $\{0,1\}^{\mathbf{Z}}$, whose unique invariant measure is not ergodic we refer the reader to page 178 of [12] (the example given in Problem 7). Of course, this example does not satisfy (1.5).

Theorem (1.4) and the first two examples above motivate two questions we have not been able to answer. In what follows we only consider translation invariant interacting particle systems on $\{0,1\}^{\mathbf{Z}^{d}}$, for which (3.1) and (3.2) hold. Under these hypotheses is it the case that $(\mathscr{I} \cap \mathscr{S})_{e} \subset \mathscr{S}_{e}$ ? Note that the first example given in this section has an important deterministic component. Since particle systems as above do not share that property, it seems plausible that for all $\mu \in \mathscr{S}, \mu S(t)$ converges. This, together with part (i) of Theorem (1.4) will imply that the answer to the above question is affirmative.

The second question is motivated by the results of $\S 5$. Is it the case that elements of $\mathscr{I}_{e} \cap \mathscr{S}$ are mixing?

Acknowledgment. I am grateful to the referee for simplifying the proof of Proposition (4.1).

\section{REFERENCES}

1. M. Aizenman (1980), Translation invariance and instability of phase coexistence in the two dimensional Ising system, Comm. Math. Phys. 73, 83-94.

2. R. L. Dobrushin (1968), The description of a random field by means of conditional probabilities and conditions of regularity, Theory Probab. Appl. 13, 197-224.

3. __ (1970), Prescribing a system of random variables by the help of conditional distributions, Theory Probab. Appl. 15, 458-486.

4. R. L. Dobrushin and S. B. Shlosman (1985), Constructive criterion for the uniqueness of Gibbs field, Statistical Physics and Dynamical Systems (J. Fritz, A. Jaffe and D. Szasz, eds), Birkhäuser, Boston, Mass., pp. 347-370.

5. E. B. Dynkin (1978), Sufficient statistics and extreme points, Ann. Probab. 6, 705-730.

6. S. N. Ethier and T. G. Kurtz (1986), Markov processes: characterization and convergence, Wiley, New York.

7. H. Föllmer (1982), A covariance estimate for Gibbs measures, J. Funct. Anal. 46, 387-395.

8. Y. Higuchi (1979), On the absence of non-translation invariant Gibbs states for the two dimensional Ising model, Colloq. Math. Soc. János Bolyai 27, 517-533.

9. Y. Higuchi and T. Shiga (1975), Some results on Markov processes of infinite lattice spin systems, J. Math. Kyoto Univ. 15, 211-229.

10. R. Holley (1971), Free energy in a Markovian model of a lattice spin system, Comm. Math. Phys. 23, 87-99.

11. R. Holley and D. Stroock (1981), Diffusions on an infinite-dimensional torus, J. Funct. Anal. 42, 29-63.

12. T. M. Liggett (1985), Interacting particle systems, Springer-Verlag, New York. 
13. N. Matloff (1977), Ergodicity conditions for a dissonant voting model, Ann. Probab. 5, 371-386.

14. D. Ruelle (1978), Thermodynamic formalism, Addison-Wesley, Reading, Mass.

IMPa, Estrada Dona Castorina 110, 22460 Rio de Janeiro, R. J., Brazil 